\title{
CIRED 2013: Bericht über Session 5 - Netzentwicklung
}

\author{
W. Tenschert OVE
}

Online publiziert am 30. November 2013

(C) Springer Verlag Wien 2013

\section{Zusammenfassung}

Die 114 Arbeiten im Bereich der Netzentwicklung von Verteilernetzen konzentrieren sich vorwiegend auf die Entwicklung von Analysemodellen für volatile Lasten und Einspeisungen, die Integration von Simulationswerkzeugen und die gemeinsame Simulation von elektro- und kommunikationstechnischen Netzen.

Zunehmende Aufmerksamkeit wird der rasant wachsenden Datenflut als Basis für Planungsaufgaben gewidmet. Insbesondere Informationen aus modernen Zählern vervielfachen die verfügbare Datenmenge. Zugehörige Bearbeitungsmethoden und -werkzeuge sind noch nicht ausreichend verfügbar. Weitere Entwicklungstätigkeit wird dringend eingefordert.

Wurden bisher deterministische Methoden für das Lastverhalten zur Erzielung robuster Lösungen angewendet, so sind vermehrt wahrscheinlichkeitsorientierte Methoden erforderlich. Langfristig zeitlich nicht planbare Erzeugungen und neue noch unbekannte Lastverläufe durch z. B. Ladevorgänge der Elektromobilität beeinflussen wesentlich den Planungsprozess für technisch und wirtschaftlich sinnvolle Netze. Traditionelle Netzauslegung auf der Grundlage von Extremfallbetrachtungen wird durch Wahrscheinlichkeitsbetrachtungen ergänzt oder ersetzt werden müssen. Zukünftig könnten Erzeugung und Last in Planungsrechnungen unter Berücksichtigung ihres zeitlichen Auftretens getrennt voneinander betrachtet werden.

Der im Verteilernetz häufig übliche Ansatz, betriebliche Erfordernisse durch die Dimensionierung und Struktur des Netzes vorweg zu lösen, wird an Grenzen wirtschaftlicher Machbarkeit stoßen. Drohenden Grenzwertverletzungen wird zukünftig auch in Verteilungsnetzen durch betriebliche Eingriffe in Form von Regelungen, Schalthandlungen oder Engpassmanagement begegnet werden müssen.

Als notwendiges Erfordernis für die Berechnung von zukünftigen Verteilernetzen wird der Begriff "cyber-physical simulation" geprägt. Die simultane Simulation von Stromnetz und Kommunikationsnetz soll deren Zusammenwirken in der Planung und im Betrieb berechenbar machen. Derzeit werden diese Analysen noch getrennt voneinander vorgenommen. Insbesondere bei Störungen in einem oder beiden Netzen kann die gegenseitige Beeinflussung zu unerwarteten und unzulässigen Effekten führen.

\section{Trends und Entwicklungen}

Quer über alle Beiträge waren auch 2013 wieder einige allgemeine Trends zu erkennen:

- Niederspannungsnetz gewinnt an Bedeutung

Die Integration von kleinen dezentralen Erzeugungseinheiten und der Elektromobilität findet in erster Linie im Niederspannungsnetz statt. Regelbare Ortsnetztransformatoren und Längsregler in der Niederspannung werden als wirksamer Lösungsansatz in vielen Beiträgen vorgestellt. Die Entwicklung von Gleichstromübertragung in der Niederspannung wird in einigen Ländern weiter verfolgt.

- Gegenseitige Abhängigkeit von Stromnetz und Kommunikationsnetz

Zeitliche Verzögerungen oder Funktionsausfälle im Kommunikationsnetz müssen für stabile Lösungen berücksichtigt werden. Analysiert werden auch die wirtschaftlichen Auswirkungen von CyberAttacken auf das Stromnetz.

- Lokale Speicher

Lokale Speicher im Mittel- und Niederspannungsnetz sind ein zentrales Thema der Forschung. Die Möglichkeit, die Auswirkung volatiler Erzeugung oder Last beeinflussen zu können, wird breit diskutiert. In der Speicherung mit unterschiedlichen Technologien und Zielfunktionen wird viel Entwicklungspotential mit wesentlichen Auswirkungen auf die Verteilernetze gesehen.

- Active System Management

Der bisher überwiegend statische Betrieb von Verteilernetzen wird zukünftig komplexer werden. Aktive Eingriffe in Erzeugung und Last im operativen Tagesgeschäft werden mit zunehmender Auslastung der Verteilernetze erforderlich sein. 22nd International Conference on Electricity Distribution, Stockholm, Schweden, 10. bis 13. Juni 2013.

Tenschert, Walter, Netz Oberösterreich GmbH, Böhmerwaldstraße 3, 4020 Linz, Österreich (E-Mail: walter.tenschert@netzgmbh.at) 